\title{
Vector design influences hepatic genotoxicity after adeno-associated virus gene therapy
}

\author{
Randy J. Chandler, ${ }^{1}$ Matthew C. LaFave, ${ }^{2}$ Gaurav K. Varshney, ${ }^{2}$ Niraj S. Trivedi, ${ }^{3}$ Nuria Carrillo-Carrasco, ${ }^{4}$ Julien S. Senac, ${ }^{1}$ \\ Weiwei Wu, ${ }^{5}$ Victoria Hoffmann, ${ }^{6}$ Abdel G. Elkahloun, ${ }^{5}$ Shawn M. Burgess, ${ }^{2}$ and Charles P. Venditti \\ 'Cenetics and Molecular Biology Branch, ${ }^{2}$ Translational and Functional Genomics Branch, and ${ }^{3}$ Computational and Statistical Genomics Branch, National Human Genome Research Institute, NIH, Bethesda \\ Maryland, USA. ${ }^{4}$ Therapeutics for Rare and Neglected Diseases, National Center for Advancing Translational Sciences, NIH, Bethesda, Maryland, USA. ${ }^{5}$ Cancer Genetics and Comparative Genomics Branch, \\ National Human Genome Research Institute, NIH, Bethesda, Maryland, USA. 'Diagnostic and Research Services Branch, Office of the Director, NIH, Bethesda, Maryland, USA.
}

\begin{abstract}
The use of adeno-associated virus (AAV) as a gene therapy vector has been approved recently for clinical use and has demonstrated efficacy in a growing number of clinical trials. However, the safety of AAV as a vector has been challenged by a single study that documented hepatocellular carcinoma (HCC) after AAV gene delivery in mice. Most studies have not noted genotoxicity following AAV-mediated gene delivery; therefore, the possibility that there is an association between AAV and HCC is controversial. Here, we performed a comprehensive study of HCC in a large number of mice following therapeutic AAV gene delivery. Using a sensitive high-throughput integration site-capture technique and global expressional analysis, we found that AAV integration into the RNA imprinted and accumulated in nucleus (Rian) locus, and the resulting overexpression of proximal microRNAs and retrotransposon-like 1 (Rt/1) were associated with HCC. In addition, we demonstrated that the AAV vector dose, enhancer/promoter selection, and the timing of gene delivery are all critical factors for determining HCC incidence after AAV gene delivery. Together, our results define aspects of AAV-mediated gene therapy that influence genotoxicity and suggest that these features should be considered for design of both safer AAV vectors and gene therapy studies.
\end{abstract}

\section{Introduction}

Adeno-associated virus (AAV) is regarded as nonpathogenic (1) and, when configured for gene therapy applications, is devoid of an intrinsic capacity to replicate and integrate. AAVs have therefore emerged as promising vectors for gene delivery (2) and have been extensively studied in small- and large-animal models for preclinical efficacy and safety (3). In fact, there are multiple human AAV gene therapy trials around the world that have been completed, are accruing participants, or are in preparation (4), and recent studies using AAV to treat 3 genetic disorders have been regarded as successful (5-8). In 2012, the first AAV treatment was approved for clinical use to treat lipoprotein lipase deficiency, a rare genetic disorder (9).

The universal safety of AAV was first questioned by a single study that described the development of hepatocellular carcinoma (HCC) in mice after systemic delivery of a therapeutic AAV gene therapy vector (10). Using a murine model of $\beta$-glucuronidase deficiency (mucopolysaccharidosis type VII), Donsante et al. documented an increased rate of HCC formation in mice treated with a therapeutic AAV vector in the neonatal period $(10,11)$. Subsequently, several larger independent investigations designed to investigate AAV-mediated genotoxicity in mice were unable to document an increased risk of tumorigenesis $(12,13)$, but these studies failed to replicate key experimental variables, such as the timing of viral administration, vector configurations, dose, delivery route,

Conflict of interest: The authors have declared that no conflict of interest exists Submitted: October 1, 2014; Accepted: December 11, 2014.

Reference information: J Clin Invest. 2015;125(2):870-880. doi:10.1172/JCI79213. and background strain susceptibility to develop HCC (14-16). Even a study that observed an increase in the development of HCCs after mice were administered AAV reporters concluded that the AAV vectors alone did not contribute to the formation of tumors; rather, Bell et al. claimed that the expression of LacZ alone or in combination with vector was causative (17). Therefore, an association between AAV and HCC in the setting of therapeutic gene delivery has remained uncertain (15-21) and mechanism(s) of AAV-mediated HCC have remained unresolved $(15,19,22)$.

In a subset of tumors from the treated mucopolysaccharidosis type VII mice, AAV integrations were tightly clustered in the RNA imprinted and accumulated in nucleus (Rian) locus on chromosome 12 (10). This is a complex genomic region that encodes numerous regulatory RNAs, including microRNAs (miRNAs), snoRNAs, and lincRNA (23). The aberrant expression of proximal small noncoding regulatory RNAs, induced by AAV vector integration, was proposed as a mechanism for carcinogenesis (10). Because the upregulation of delta-like homolog 1-deiodinase type 3 (DLK1-DIO3), the orthologous genomic imprinted cluster of Rian locus microRNAs found in humans, has been associated with poor survival in patients with hepatic carcinoma (24), the further delineation of potential AAV genotoxicity, especially in the setting of systemic or liver-directed gene therapy is mandated.

Our previous studies using neonatal AAV gene delivery to treat mice with methylmalonic acidemia (MMA) provided a large set of homogenously treated animals that were used to interrogate the possible genotoxicity of AAV gene therapy and explore mechanisms of carcinogenesis. We had demonstrated that AAV serotype 2, 8, and 9 vectors configured to express therapeutic 
Table 1. Frequency of HCC in control and AAV-treated mice followed for 18 to 22 months for various AAV vectors

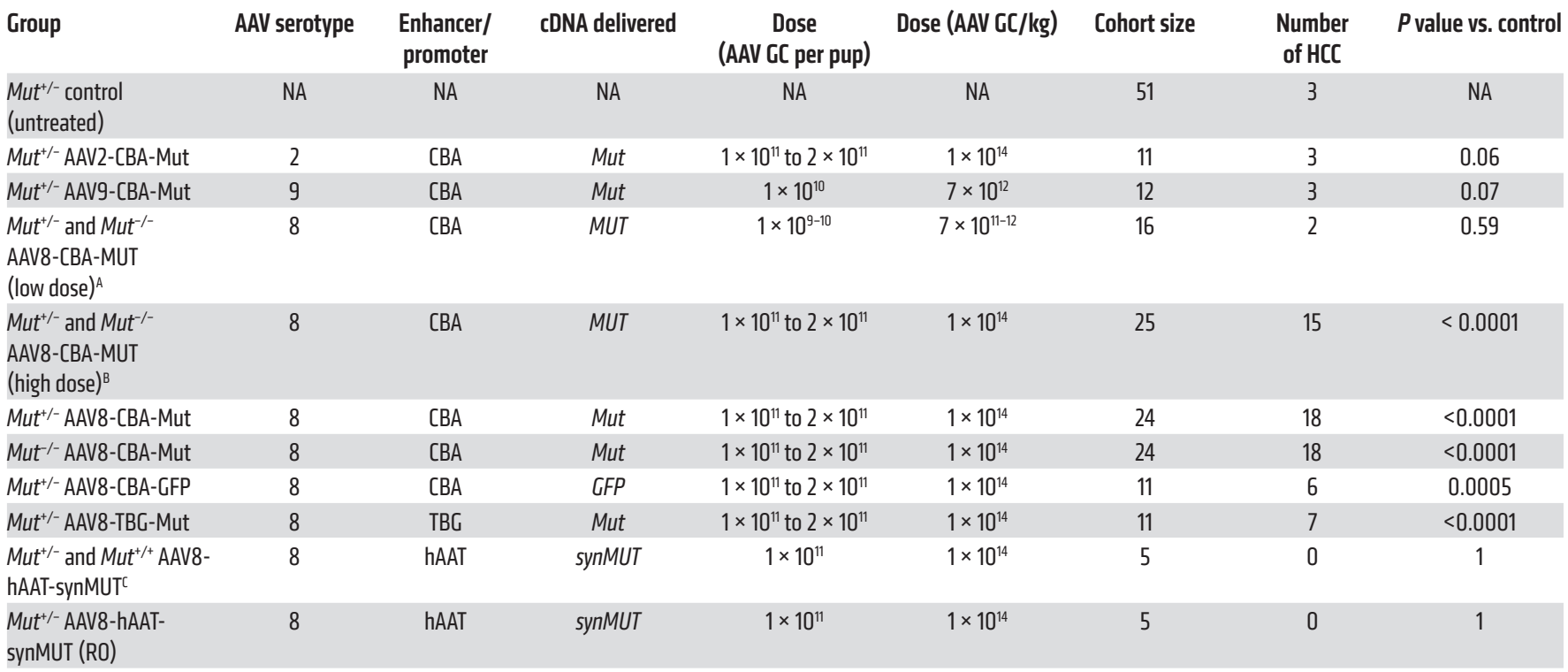

AAV delivered in the neonatal period (also see Figure 1). AAV was delivered via intrahepatic injection unless retro-orbital (RO) injection is indicated in the group column. $P$ values were calculated using Fisher's exact test (2 tailed). ${ }^{A}$ This cohort contains mice treated with $1 \times 10^{9} \mathrm{GC}$ per pup (6 Mut ${ }^{+/-}$mice [0 HCC]) and $1 \times 10^{10} \mathrm{GC}$ per pup $\left(5 \mathrm{Mut}^{+/-}\right.$mice $[1 \mathrm{HCC}]$ and $5 \mathrm{Mut}^{-/-}$mice $\left.[1 \mathrm{HCC}]\right)$. ${ }^{\mathrm{B}}$ This cohort contains mice treated with $1 \times 10^{11}$ to $2 \times 10^{11} \mathrm{GC}$ per pup $\left(13 \mathrm{Mut}{ }^{+/-}\right.$mice [6 HCCs] and $12 \mathrm{Mut}^{-/-}$mice [9 HCCs]). ${ }^{~}$ This cohort contains 5 mice $\left(3 \mathrm{Mut}^{+/-}\right.$and $\left.2 \mathrm{Mut}^{+/+}\right)$.

transgenes were highly effective at rescuing the neonatal lethal phenotype displayed by mice with MMA (25-28). In the present studies, these large cohorts of treated and control mice were followed for 22 months to monitor the efficacy of neonatal AAV gene therapy and survey for long-term complications. We observed a significant increase in the incidence of HCC, as high as $75 \%$ in some groups, after AAV gene delivery and used a novel high-throughput integration site-capture technique to locate AAV insertions in tumors and matched control tissues, coupled with expression analysis, to interrogate the mechanism(s) of viral genotoxicity (29). Although the highly expressed, liver-specific genes albumin and $\alpha$-fetoprotein emerged as the most frequent targets for AAV integration in both healthy livers and HCCs, only vector-mediated insertional mutagenesis into the Rian locus was associated with HCC following gene therapy. HCC formation depended upon the vector dose and was eliminated by changing the enhancer promoter of the vector. Our studies help elucidate the mechanisms underlying AAV-mediated genotoxicity in mice and provide a rational monitoring approach to assess the safety of AAV vectors in preclinical models as well as human subjects.

\section{Results}

$A A V$ gene therapy confers an increased risk of developing HCC independent of mouse genotype, viral transgene, or genetic background. AAV vectors expressing a therapeutic murine methylmalonylCoA mutase cDNA ( $M u t)$, human methylmalonyl-CoA mutase cDNA (MUT), or GFP transgene under the control of the ubiquitous chicken $\beta$-actin (CBA) promoter (AAV serotypes 2, 8, and 9) or the liver-specific thyroxine-binding globulin (TBG) promoter (AAV serotype 8 ) were administered to MMA and control mice in the immediate neonatal period (Figure $1 \mathrm{~A}$ ). Control and AAVtreated mice were derived using the same sets of breeding pairs and were genetically similar, predominantly consisting of the C57BL/6 background (25). All untreated $\mathrm{Mut}^{-/-}$mice died in the neonatal period. In the cohort of animals that had received a single intrahepatic injection of $1 \times 10^{11}$ or $2 \times 10^{11}$ genome copies (GC) $\left(\sim 1 \times 10^{14} \mathrm{GC} / \mathrm{kg}\right)$ of an AAV8-CBA-Mut vector (25), HCCs were detected at between 12 and 21 months in greater than $70 \%$ of both $M u t^{+/-}$and $\mathrm{Mut}^{-/-}$mice $(n=48)$ (Figure 1B and Table 1). No sex differences in the incidence of cancer were observed, with $82 \%$ of the male mice $(n=22)$ and $69 \%$ of female mice $(n=26)$ developing HCC following AAV treatment $(P=0.5048$, Fisher's exact test). The discovery of HCC was often preceded by the appearance of a nontender abdominal mass, followed by weight loss. The tumors were multinodular and displayed typical morphological and histopathological characteristics of HCC (Supplemental Figure 1, A-G; supplemental material available online with this article; doi:10.1172/JCI79213DS1).

A second group of $11 \mathrm{Mut}^{+/-}$mice was administered $1 \times 10^{11} \mathrm{GC}$ of AAV8-CBA-GFP, delivered in an identical manner. Greater than $50 \%$ of the mice in this cohort developed HCC at between 14 and 25 months after the delivery of the GFP reporter transgene (Figure 1B). There was no statistically significant difference in the incidence of HCC between the AAV8-CBA-Mut-treated mice of either genotype compared with the mice that received AAV8-CBA-GFP (Table 1). These results indicate that the development of HCC is not associated with a particular transgene.

In contrast to the AAV-treated cohorts, a control group of untreated $\mathrm{Mut}^{+/-}$mice $(n=51)$ was followed in parallel for 18 to 25 months, with only 3 mice developing HCC $(<10 \%)$ (Figure $1 \mathrm{~B}$ and 
A AAV8-CBA-Mut, AAV2-CBA-Mut and AAV9-CBA-Mut

ITR CMV promoter enhancer

ITR CMV promoter enhancer

ITR

ITR AMBP promoter enhancer 1 TBG promoter

AAV8-hAAT-synMUT
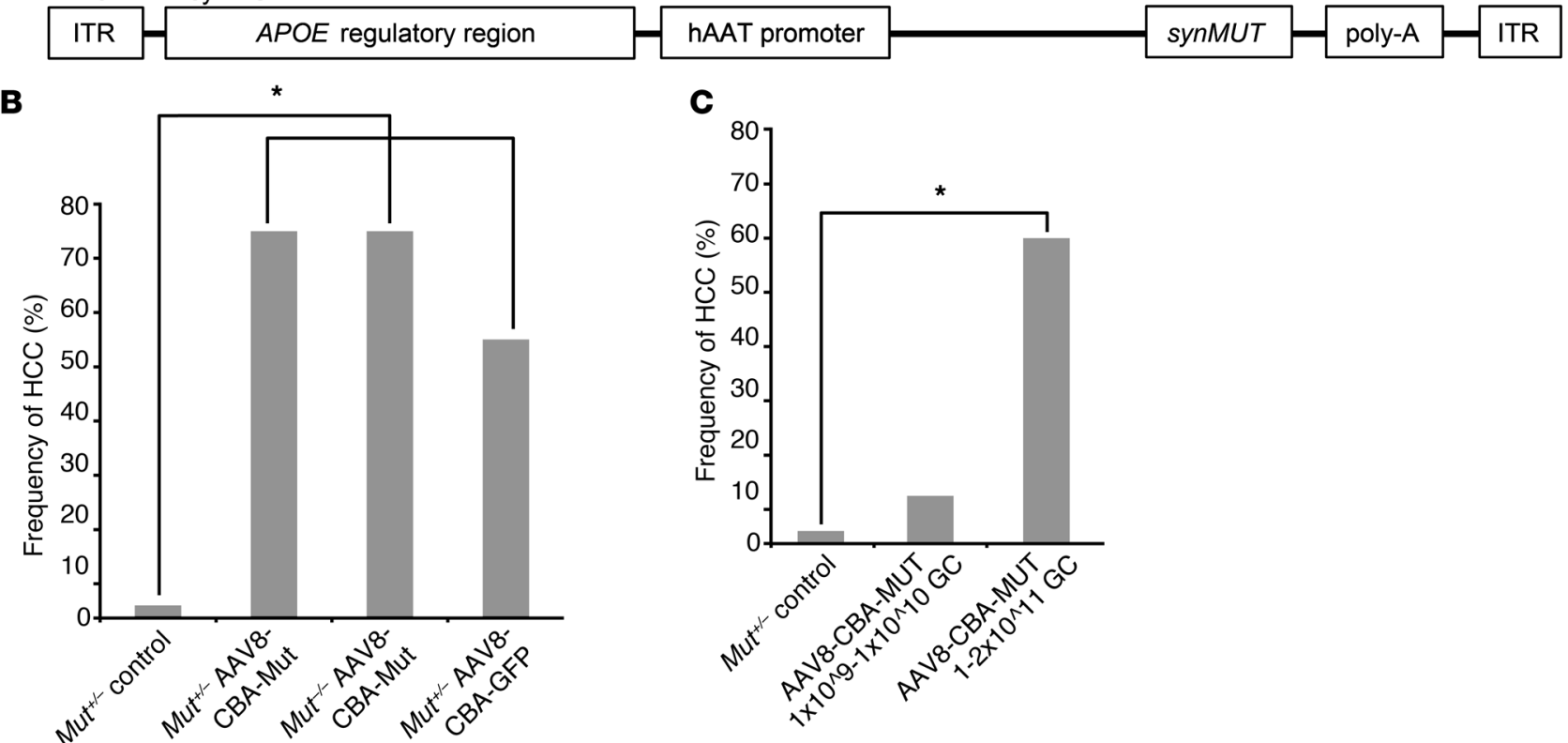

Figure 1. Incidence of HCC in mice followed for $\mathbf{1 8}$ to $\mathbf{2 2}$ months after intrahepatic neonatal injection of AAV vectors. (A) Schematic of AAV vectors packaged into AAV serotypes 2, 8, and 9 and used for gene delivery. AMBP, $\alpha$-1-microglobulin; APOE, apolipoprotein E; ITR, AAV2 ITR; TBG, human TBG promoter. (B) Contribution of the murine background and transgene expression to the frequency of HCC. Untreated Mut ${ }^{+/-}$control mice $(n=51)$ were aged for 22 months and, at death, were assessed for hepatic carcinoma. Three HCCs were detected in this group. Mut ${ }^{+/-}(n=24)$ and Mut ${ }^{-/-}(n=24)$ mice were treated with $1 \times 10^{11}$ to $2 \times 10^{11} \mathrm{GC}$ per pup of AAV8-CBA-Mut, and Mut ${ }^{+/-}$mice $(n=11)$ were treated with $1 \times 10^{11}$ to $2 \times 10^{11} \mathrm{GC}$ per pup of AAV8-CBA-GFP. All untreated Mut $^{{ }^{-/}-}$mice perished in the newborn period. (C) Relationship between AAV dose and the frequency of HCC following injection. Untreated Mut ${ }^{+/-}$control mice $(n=51)$ compared with Mut $t^{+-}$and Mut ${ }^{-/-}$mice treated with AAV8-CBA-MUT at doses of $1 \times 10^{9-10} \mathrm{GC}(n=16)$ or $1 \times 10^{11}$ to $2 \times 10^{11} \mathrm{CC}(n=25)$ in the neonatal period. (See also Table 1.) ${ }^{*} P<0.01$ (Fisher's exact test, 2 tailed).

Table 1). In aggregate, these results demonstrate that the increased rate of tumorigenesis seen in the animals that received AAV was not dependent upon the $M u t$ genotype, the vector transgene, or genetic predisposition.

HCC incidence is $A A V$ dose dependent. We next performed a preclinical AAV dose reduction study in attempt to determine the minimal dose needed to rescue the MMA mice (28). We subsequently found that reducing the AAV8 dose from $1 \times 10^{11}$ to $2 \times 10^{11} \mathrm{GC}$ (approximately $1 \times 10^{14} \mathrm{GC} / \mathrm{kg}$ ) to $1 \times 10^{9-10} \mathrm{GC}(7$ $\times 10^{11-12} \mathrm{GC} / \mathrm{kg}$ ) decreased the aggregate incidence of HCC at 22 months from $84 \%$ to $12 \%$ (Figure $1 \mathrm{C}$ and Table 1). While the lower dose resulted in an increased frequency of HCC formation compared with the untreated controls, it was not significant (Table 1). The dose-toxicity correlation we observed following intrahepatic AAV delivery with identical volumes and the fact that systemic delivery of vector has been associated previously with AAV HCC formation in mice $(11,17)$ suggests that intrahe- patic delivery is likely not a major factor influencing HCC development in our studies.

Genes highly expressed in the liver are common targets for $A A V$ integration. To investigate the possibility that insertional mutagenesis by AAV contributed to the development of HCC, we adapted an established integration capture method with subsequent high-throughput sequencing (29) to characterize AAV integrations. We identified and mapped 2,834 unique AAV integrations in 33 HCCs and 31 matched control livers (Figure 2A and Supplemental Table 1). A majority of the genes lacked integrations, and those genes with integrations typically harbored a single unique AAV integration. However, genes known to have higher expression in the neonatal liver, specifically albumin and $\alpha$-fetoprotein, were the most susceptible to AAV integrations (Figure 2). The integrations in these 2 loci were equally distributed between the HCC and control livers and therefore were not associated with HCC formation. The preference for AAV integration into active genes 


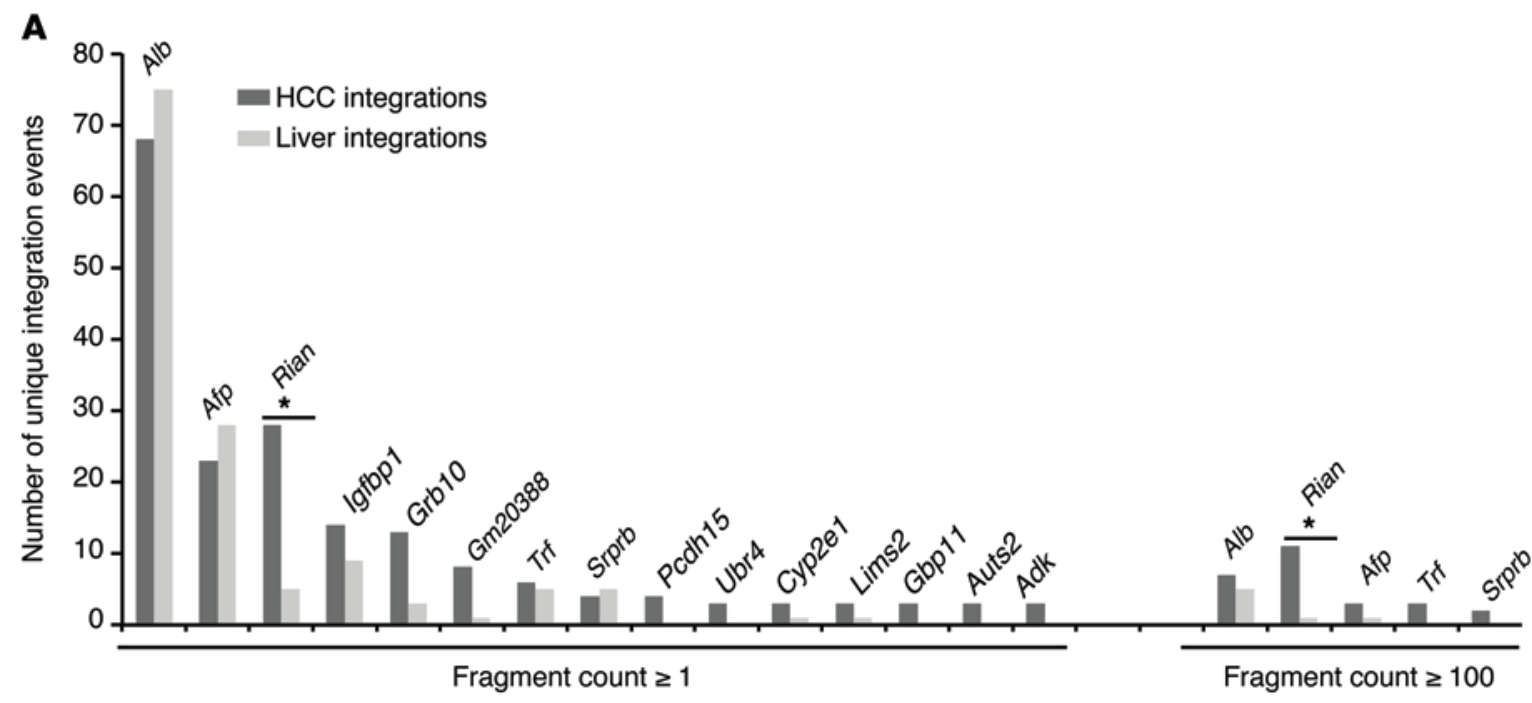

B
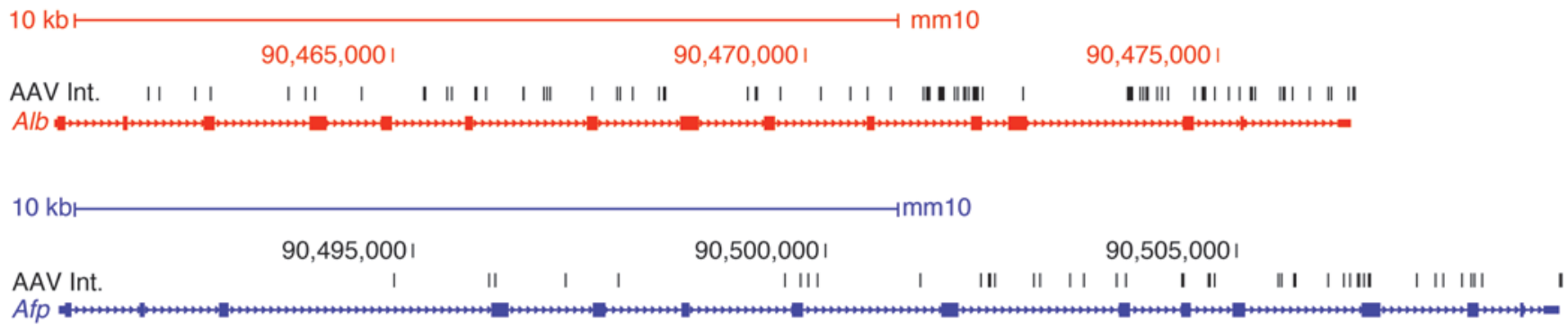

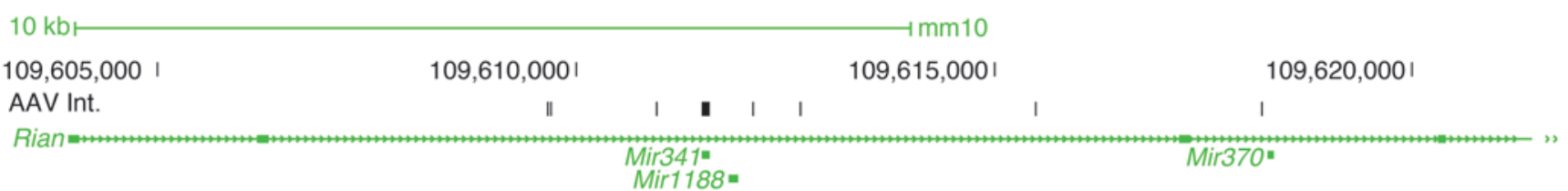

Figure 2. Vector integration profile in normal livers and HCCs after AAV delivery. (A) Genes with 3 or more independent AAV integrations identified by integration capture and subsequent high-throughput sequencing in HCCs $(n=33)$ in comparison to integrations in normal livers $(n=31)$. The fragment count is equal to the number of times a unique integration sequence is detected. Due to size constraints, fragment count $\geq 1$ shows all genes with 3 or more unique integrations. Fragment counts $\geq 100$ are filtered for genes with more strongly amplified integration sites. ${ }^{*} P<0.01$ (Fisher's exact test, 2 tailed). (B) Integrations of AAV8-CBA-Mut, AAV8-CBA-MUT, and AAV8-TBG-Mut vectors into the albumin (red), $\alpha$-fetoprotein (blue), and Rian (green) loci. Each gene is depicted with exons in solid black, with solid lines representing an independent integration event. Thicker lines indicate multiple AAV integrations. The thick line over the Rian locus is created by the 12 independent HCC-associated integrations that map within a small genomic window. (See also Supplemental Table 1.)

has been described previously (30) and likely contributes to the enrichment observed at these loci.

Vector integration into the Rian locus is significantly increased in HCCs after AAV gene delivery. As shown below, only the Rian locus exhibited a greater number of integrations, with higher fragment counts in the HCCs compared with those in adjacent control tissue (Figure 2A). The fragment count is the number of times a unique sequence is detected, and it was used to approximate the copy number of a specific AAV integration. There were 29 unique AAV integrations in the Rian locus identified in 21 independent HCCs and only 5 unique AAV integrations, with a fragment count of 1 or greater, in the Rian locus identified in 5 normal appearing liver samples obtained from mice with HCC (Figure 2A). AAV integrations that drive HCC formation might be selected and therefore present at a higher copy number than noncausative integrations in the tumors. Consistent with this hypothesis, only AAV integrations in Rian were increased in HCCs compared with those in normal livers at a fragment count of 100 or greater.

A majority of the AAV integrations in Rian were located in Mir341, a microRNA with a 96-base pair coding region contained within the Rian locus (Figure 3A). We confirmed that AAV integration junctions in the Rian locus in 15 independent HCCs were tumor specific and clonal by independently amplifying each junction fragment, sequencing the resultant products, and assessing the relative copy number (Figure $3 \mathrm{~B}$ and Supplemental Table 2). The junction fragments were present only in the HCCs and were not detectable in normal liver tissue dissected from the tumor margins of the same mice (Figure 3B).

Upregulation of noncoding RNAs and genes proximal to Rian in $A A V$-associated HCCs. We performed expression profiling of the 


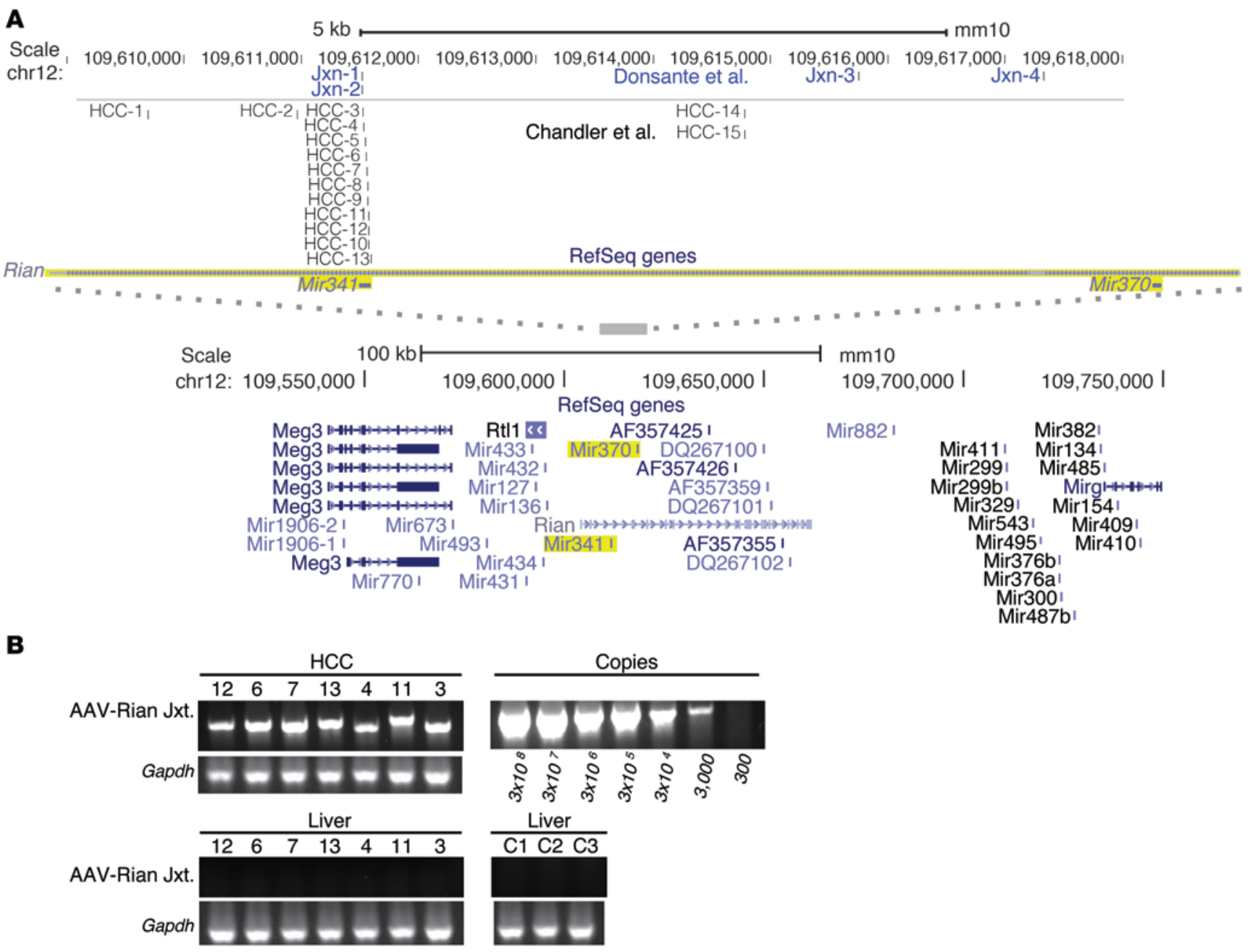

Figure 3. Vector integrations in AAV-associated HCCs. (A) Genomic locations (UCSC Genome Browser, Genome Reference Consortium Mouse Build 38 December 2012, mm10) of HCC integration junctions validated after PCR amplification and Sanger sequencing compared with HCC integration junctions identified previously by Donsante et al (9). (See also Supplemental Tables 1 and 2.) The bolded gene, Rt/1, and microRNAs were increased in expression in the HCCs. (B) Within the Rian locus, semiquantitative targeted PCR amplification of common AAV vector-Mir341 junctions in both HCCs and adjacent normal livers from the same mice and mice (C1-C3) that were treated with AAV but did not develop cancer. The numbers above the HCC lanes refer to the HCC integration junctions presented in A. Comparison to vector amplification standards (left) suggests the integration junctions are present an average of approximately 1 copy per hepatocyte.

HCCs compared with that of controls using both global RNA- and microRNA-specific microarrays. The global expression analysis compared HCC to normal liver tissue, and tumor-free margins displayed a distinct pattern with the HCCs clustering tightly into a separable group (Figure 4A). There was a marked upregulation of genes near AAV integrations in Rian in the HCCs (Figure 3A, Figure 4A, and Supplemental Table 3). In addition, a unique subset of aberrantly expressed coding and noncoding RNAs, representing potential biomarkers and therapeutic targets for HCC, also emerged from these studies (Figure 4 and Supplemental Table 3). The overexpression of one gene in proximity to the AAV-Rian integrations, retrotransposon-like 1 ( $R t l 1)$, a retrotransposon-derived gene with a predicted transmembrane domain and protease activity (31), was most apparent in our initial microarray experiment (Figure 4B and Supplemental Table 3). We next used microRNA-specific microarrays to better characterize the microRNA expression profile of the HCCs (Figure 4C and Supplemental
Tables 3 and 4) and confirmed the upregulation of Rian-encoded microRNAs in the AAV HCCs as well as the downregulation of distinct, unlinked miRNAs on chromosome 4. Finally, we independently confirmed the overexpression of Rtl1 and Mir543 in the HCCs using qPCR (Supplemental Tables 4 and 5).

Enhancer promoter selection plays a role in HCC development after AAV gene delivery. We observed variable but increased rates of HCC in mice treated with AAVs of differing serotypes and enhancer promoters (Figure 5A and Table 1). Our results (Figure $5 \mathrm{~A}$ and Table 1) and those previously reported document HCCs in mice after receiving AAV vectors carrying the TBG and CBA enhancer promoters $(11,17,32)$ and not in mice after receiving AAVs configured with other enhancer promoters (13). Therefore, we hypothesized that the trans regulatory sequences carried by the vector were influencing AAV genotoxicity.

To test this hypothesis, we designed an AAV using a human $\alpha-1$ antitrypsin (hAAT) promoter, similar to the promoter used in 


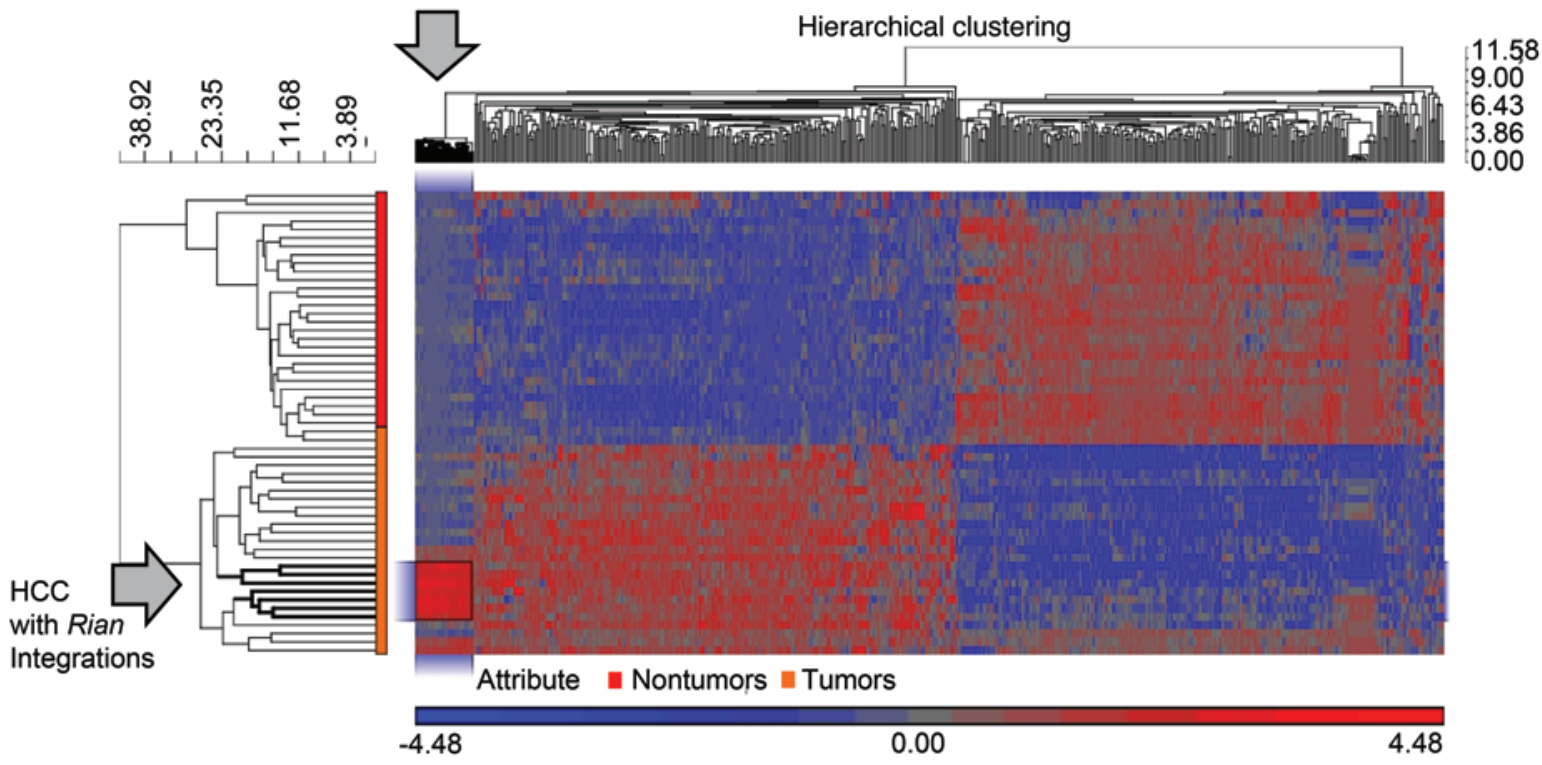

B

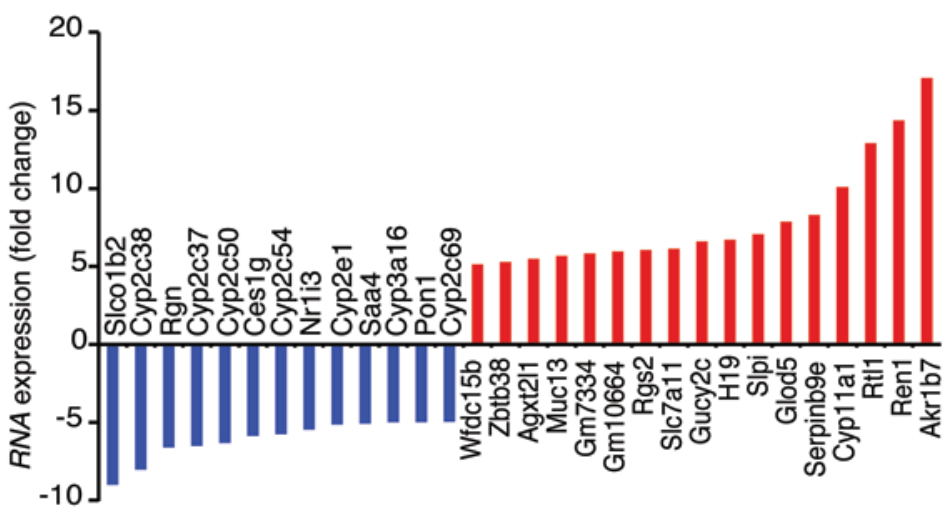

C

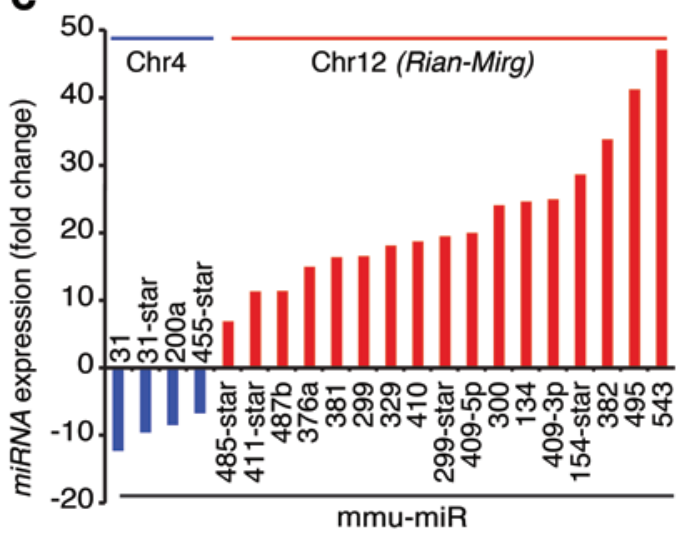

Figure 4. Global RNA and miRNA expression profiles of HCC versus normal liver tissue. (A) Dendrogram displays the hierarchical clustering of genes with similar expression patterns. Red indicates an increase and blue indicates a decrease in gene expression in the HCC versus normal liver tissue. The scale (red to blue) represents the ratios of differential expression based on signal intensity, while the numbers to the right and left denote the Euclidean sample dissimilarity (Partek Genomic Suite). (B) RNA expression changes of 5-fold or greater in HCCs $(n=29)$ relative to normal liver tissue $(n=28)$ detected with RNA microarray. (C) miRNA expression changes of 5-fold or greater in HCCs $(n=12)$ relative to normal liver tissue $(n=6)$ detected with miRNA-specific microarray. $P<0.01$ for all changes in expression (ANOVA). (See also Supplemental Tables 3-5.)

human factor IX gene therapy clinical trials $(7,33)$, to drive the expression of a codon-optimized human MUT cDNA (synMUT) (Figure 1A). $\mathrm{Mut}^{+/-}$and $\mathrm{Mut}^{+/+}$mice were treated in the immediate neonatal period with AAV8-hAAT-synMUT at a dose of $1 \times 10^{11} \mathrm{GC}$ (approximately $\left.1 \times 10^{14} \mathrm{GC} / \mathrm{kg}\right)$ via systemic $\left(\mathrm{Mut}^{+/-}\right.$ mice, $n=5$ ) or direct hepatic ( $3 \mathrm{Mut}^{+/-}$and $2 \mathrm{Mut}^{+/+}$mice) injection (Figure 5A and Table 1).

After 18 to 22 months, none of the 10 AAV8-hAAT-synMUTtreated animals developed HCC (Figure 5A). A subsequent integration analysis using 5 livers from the mice that received direct hepatic injections of vector identified 3,898 unique AAV integrations (Supplemental Table 6). These livers were normal in gross appearance, without nodules, and had normal histology. As observed with the AAV8-CBA-Mut, AAV8-CBA-MUT, and AAV8-TBG-Mut vectors (Figure 2B), the albumin, $\alpha$-fetoprotein, and Rian genes were frequent integration loci (Figure $5 \mathrm{~B}$ ). We detected 6 unique AAV integrations at the Rian locus in the 5 livers studied (Figure 5, B and C). The AAV8-hAAT-synMUT Rian integrations clustered in the same genomic region, very close to Mir341, as seen with the other AAV vectors. In contrast to the integration patterns of other AAV vectors studied, AAV8-hAATsynMUT showed a distinct set of loci, with both low and high fragment counts (Figure 5B and Supplemental Table 6).

\section{Discussion}

We observed a dramatic, dose-dependent increase in HCC incidence in a large number of mice following neonatal AAV delivery that was not dependent upon the murine genotypes or the viral transgene. Although hepatic perturbations have been well documented in mice and patients with MMA $(34,35)$, neither the disease state nor carrier status for MMA is recognized to confer an increased risk to develop HCC. Our observations 

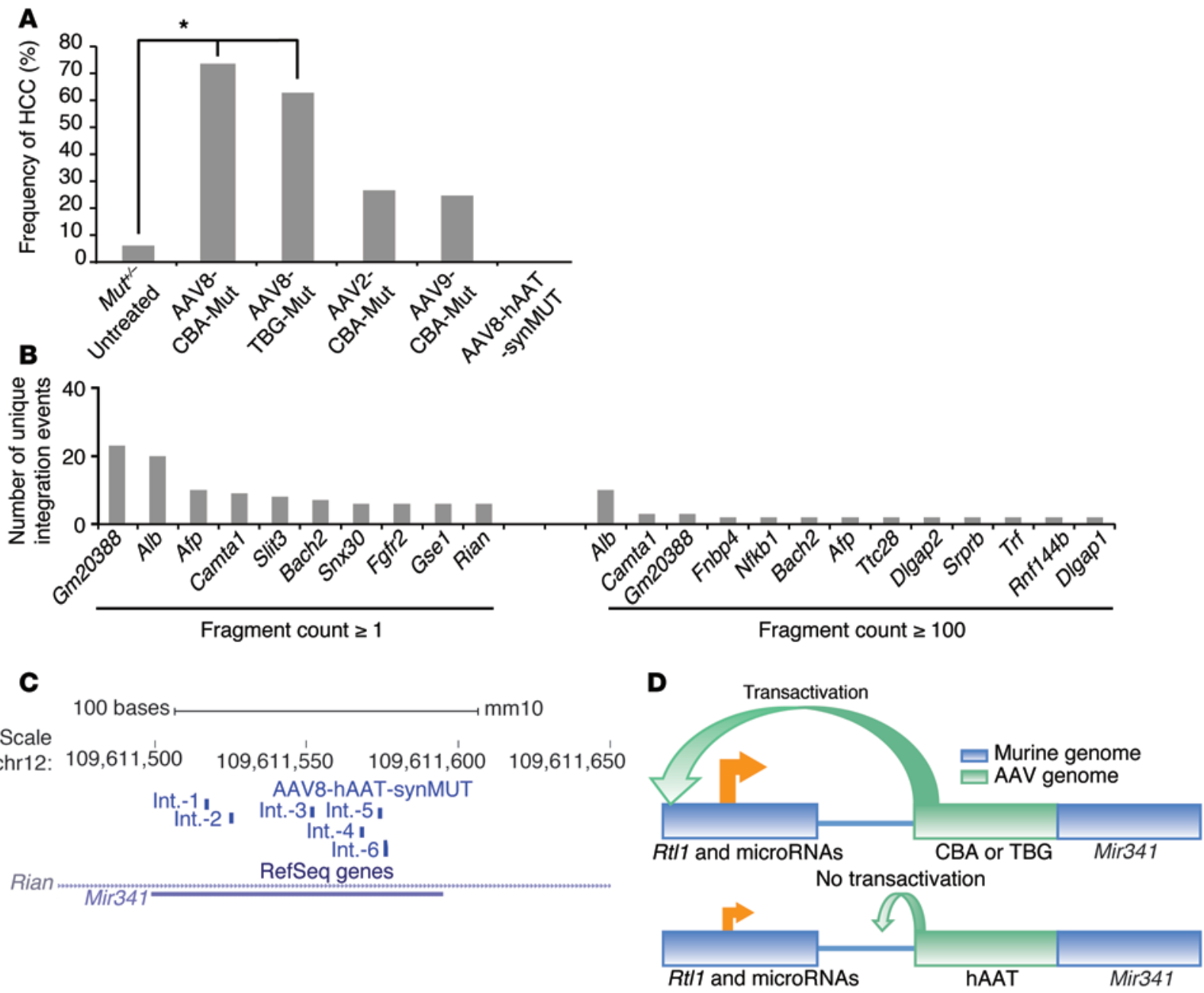

Figure 5. Regulatory elements influence HCC incidence after AAV gene delivery. (A) Frequency of HCC in mice when treated with different AAV serotypes and enhancer promoter elements in comparison to that of uninjected $\mathrm{Mut}^{+/-}$control mice $(n=51)$. Mut $\mathrm{t}^{+/-}$mice treated with AAV8-CBA-Mut $(n=24)$, AAV8-TBG-Mut ( $n=11)$, and AAV2-CBA-Mut $(n=11)$ at a dose of $1 \times 10^{11}$ to $2 \times 10^{11} \mathrm{GC}$ per pup, AAV9-CBA-Mut $(n=12)$ at a dose of $1 \times 10^{10} \mathrm{GC}$ per pup, and AAV8-hAAT-synMUT at a dose of $1 \times 10^{11} \mathrm{GC}$ per pup $\left(n=10,8 \mathrm{Mut}^{+/-}\right.$and $2 \mathrm{Mut}^{+/+}$mice). (See also Table 1.) ${ }^{*} P<0.01$ (Fisher's exact test, 2 tailed). (B) Livers from mice $\left(n=5 ; 3 \mathrm{Mut}^{+/-}\right.$and $2 \mathrm{Mut}^{+/+}$mice) treated with AAV8-hAAT-synMUT after direct hepatic injection were used to characterize vector integrations. The fragment count is equal to the number of times a unique integration sequence is detected. Fragment count $\geq 1$ shows genes with the greatest number of unique integrations. Fragment counts $\geq 100$ are filtered for genes with more strongly amplified integration sites. (See also Supplemental Table 6.) (C) Six unique AAV integrations detected at 18 months of age in the normal appearing livers of 3 of 5 treated mice. (D) Schematic of hypothesis. CBA and TBG, but not the hAAT promoter enhancer, are capable of promoting increased transcription of proximal genes (transactivation) that drive the formation of HCC. The orientation of the vector integration events was not determined and therefore remains hypothetical.

offer the most compelling evidence to date that therapeutic gene delivery mediated by AAV can display marked genotoxicity.

In contrast to other preclinical studies $(16,17)$, the untreated control heterozygous mice used in this work did not display a strain tendency toward the development of HCC (Figure 1B and Table 1). The rate of HCC observed in our mice, which had contributions from C57BL/6J, 129Sv/Ev and FVB/N strains, is consistent with previously reported rates of HCC in mice with similar strain backgrounds. Several studies are relevant along these lines. Jeganathan et al. (36) documented a spontaneous HCC formation rate of $14 \%$ in a control cohort of 170 mice on a mixed C57BL/6J $\times 129 \mathrm{~Sv} / \mathrm{Ev}$ background that were aged for over 30 months. The incidence of spontaneous hepatic tumors arising in FVB/N mice appears similar, with approximately $20 \%$ of male mice $(n=29)$ developing hepatic tumors by the age of 24 months (37). C57BL/6J mice seem relatively more resistant to HCC than other strains, with $8.8 \%$ of a cohort aged 22-27 months developing hepatic cancer (38). We measured a spontaneous HCC rate of $\sim 6 \%$ (3 mice with HCC in 51 mice) in the Mut heterozygote mice used as controls, which is close to, but below, the rates other groups have documented in the respective mixed or pure strains, suggesting that a heterozygote predisposition to HCC is unlikely.

The use of an improved method to capture and characterize AAV integrations afforded the opportunity to not only make important observations about the integration profiles observed in the setting of therapeutic gene delivery but to probe mechanisms of genotoxicity in AAV-associated HCCs. Although linear amplification-mediated PCR and ligation-mediated PCR-based (LM-PCR-based) capture methods can fail to retrieve integration sites where the AAV's inverted terminal repeats (ITR) 
remain intact, the use of LM-PCR with 3 parallel frequent-cutting enzyme digests (MseI, BfaI, and Csp6I) has provided an improved recovery, fewer artifacts, and lower amplification distortion in studies using this method to capture retroviral integrations (29) and was therefore adapted to the study of AAV. We found more unique AAV integrations in the albumin and $\alpha$-fetoprotein loci, which are both highly expressed in the liver in early life, in comparison to other genes with integrations. These genes were found to have a disproportionate number of AAV integrations for all vectors and serotypes tested. The pattern of loci-harboring AAV8-hAAT-synMUT integrations was distinct, but overlapping, with what was observed with other AAV vectors, indicating that the sequence of the AAV genome delivered may also influence integration preference.

The temporal expression of $\alpha$-fetoprotein and the neonatal delivery of AAV most likely explain the integration preference for this locus, supporting the suggestion that the level of expression at an endogenous locus at the time of gene delivery is an influential factor in determining where an AAV vector integration may occur (30). It is noteworthy that the Afp and Alb genes are tightly linked, which suggests that open chromatin, in the setting of active transcription and the vector itself, presents interacting factors that influence where an AAV vector integrates (39). While AAV is known to have a preference to integrate into actively transcribed genes (30), we are unaware of any reports that correlate higher tissue-specific gene expression with increased AAV integration frequency.

We found that AAV insertions in Rian were associated with HCC. Insertional mutagenesis by AAV has been reported only after neonatal gene delivery and not after AAV administration to juvenile (6-8 weeks) (12) or older mice (13). While there have been several studies examining HCCs in mice exposed to AAV, the few that have confirmed integrations in the tumors recovered a very small number of integrations (15 total from 22 HCCs analyzed) that were dispersed in a few proto-oncogenes and tumor suppressors (16) or characterized integration events in a single control and 2 HCCs derived from mice initially claimed to not develop HCC after AAV gene therapy (20). While the latter study used a high-throughput sequencing LM-PCR capture method, Rian integrations were detected in both the control and HCCs, preventing the authors from concluding that these rAAV integration events marked hepatocytes for progression into nodules or tumors (20). A more comprehensive study was performed by Li et al., who treated adult wild-type C57BL/6 mice with a single-stranded AAV vector that expressed factor IX under the control of the hAAT promoter. These authors documented low-frequency integrations in albumin (Supplemental Table 1 in ref. 13) but not in $\alpha$-fetoprotein or Rian (13). In fact, Li et al. recovered $8 \mathrm{AAV}$ integrations (6 in HCC and 2 adjacent normal tissue) in the Alb gene; 3 match integrations we detected in mice treated with the AAV8-CBA-Mut AAV vector (Supplemental Table 4). The absence of Rian integrations in adult mice treated with AAV could be explained by the fact that Rian is expressed at higher levels in the livers of neonatal mice compared with that in those of the adult mice (40) and that AAV demonstrates a preference to integrate into actively transcribed genes (30). Therefore, the genotoxicity we observed appears to be the consequence of AAV gene delivery at a time point when the Rian locus, which is highly susceptible to insertional mutagenesis (32, $41,42)$, is vulnerable to AAV integrations $(43,44)$.

Many of the AAV integrations in Rian clustered very tightly in the Mir341 locus, which lacks a human ortholog. No microhomology between any AAV vector genome and the integration sites in Mir341 could be detected. Because we were unable to capture both ends of the integration events and the primers were anchored within the ITR, we, as well as others (10), were unable to determine the orientation of the AAV genome with respect to the Rian locus. Our integration data (Figure 2A) suggest either a strong local preference for integrations in Rian/Mir341 or increased detection of this locus as a result of selection and clonal expansion. It seems likely that both factors are contributory.

In addition to the upregulation of proximal microRNAs at the Rian locus in the AAV HCCs, we identified a gene, Rtl1, with increased expression that had not been noted previously by Donsante et al. (10). The overexpression of Rtl1 in vivo, as an independent transgene, has been shown to promote HCC formation in mice with high penetrance (31). Furthermore, increased expression of Rtl1 after lentiviral insertion into the Rian locus has been shown to drive hepatocellular carcinogenesis (42). These findings support the concept that the upregulation of $R t l 1$ caused by AAV integration into the Rian locus, as well as the aberrant expression of numerous microRNAs, is the cause of HCC in a majority of the AAV-treated mice. In addition to genes that appear locally upregulated by AAV integrations, the large number of differentially expressed genes we identified in the cancers (Figure 4), presumably representative of state of HCC itself, may inform the future study, diagnosis, and treatment of HCC.

Like other gene therapy vectors, the enhancer promoter of the AAV transgene appears to play a crucial role in tumorigenesis (32). Regulatory elements within the sleeping beauty transposon have been shown to influence insertional mutagenesis (41). Given that HCC was only associated with AAV gene therapy using either CBA or TBG enhancer promoters, we speculated that vector-encoded cis regulatory sequences were contributory. To investigate this possibility, we developed a vector, AAV8-hAATsynMUT, modeled on a AAV vector that had been administered to human subjects with hemophilia and did not cause genotoxicity in mice $(13,33)$. While we did not observe HCC in mice treated with AAV8-hAAT-synMUT, we did detect AAV integrations in Rian in the livers of treated mice, indicating that this locus is a site of AAV integration and is detectable in the absence of clonal selection. The expression of Rtl1 and Mir543 was not found to be upregulated in the livers of AAV8-hAAT-synMUT-treated mice (Supplemental Table 4), suggesting that localized transcriptional upregulation secondary to AAV8-hAAT-synMUT integration was not present. We postulate that the integrations did not predispose to HCC, because the hAAT enhancer promoter, unlike the TBG and CBA regulatory elements, did not lead to the overexpression of local genes. The fact that other investigators used a similar cassette to deliver the factor IX gene to 8-week-old mice but did not detect integrations into the Rian locus (13) may be explained by the differing ages of delivery between the 2 cohorts.

Although experiments in small animals do not always accurately predict clinical outcomes, the genotoxicity we have observed with various AAV vectors warrants concern about the 
possibility of insertional mutagenesis following AAV gene delivery in humans. The most relevant example is the failure of animal studies to predict the increased risk for the development of leukemia after retroviral gene therapy for X-linked severe combined immunity deficiency (45). Mir341, the locus we found to be susceptible to insertion mutagenesis in mice, has no human homolog and therefore itself could not be a target of AAV integrations in human subjects. Our observations show that the species genome and vector integration profiles play import roles in determining whether AAV integrations manifest in genotoxicity and highlight the difficulties of relying heavily upon animal toxicology studies to predict human safety outcomes.

The data from this report and others (46) have implications not only for the design of AAV vectors for use in humans but for improved monitoring strategies in preclinical gene therapy experiments. While cassettes with strong enhancers and promoters, such as those that contain viral transcriptional control sequences, may display impressive preclinical efficacy, the data presented here highlight the malignancy risk that accompanies unintended integrations with subsequent cis activation. It is worth emphasizing that the genome configuration of all the AAV vectors studied here was single-stranded because of the packaging capacity limitations imposed by a double-stranded or self-complementary design. Self-complementary AAV vectors are claimed to exhibit superior in vivo efficacy compared with that of single-stranded vectors (47), but whether the self-complementary genome configuration influences integration preference compared with an equivalent single-stranded AAV vector is unknown and worthy of comparative study. Our data suggest that augmenting hepatocyte transduction by either increasing the dose or the use of a more hepatotrophic serotype, such as AAV8 compared with AAV2, led to an increase in the rate of HCC. Additionally, the use of distinct capsids to pseudotype AAV vectors (48), such as serotypes 8 and 9 , confers distinct patterns of tissue trophism in wild-type and disease states (49). Other capsid-specific properties may influence genotoxicity, such as subcellular localization (50), uncoating kinetics (51), and perhaps even transcription of the input genome (52). Preclinical testing should therefore be designed to account for the differential behavior of each vector-capsid uniquely and incorporate long-term observation for malignancies as well as genomic analyses to characterize vector integrations.

In conclusion, we have demonstrated that AAV administration can predispose to HCC formation. There are multiple variables influencing AAV-mediated insertional mutagenesis and subsequent genotoxicity, including the age of treatment, dose, serotype, enhancer promoter encoded by the vector, and the species genome. The fact that AAV cassette design can markedly alter the incidence of genotoxicity is encouraging and illustrates how a more complete understanding of vector behavior in preclinical models could inform the risks of potential adverse events associated with AAV gene delivery in humans.

\section{Methods}

Murine model of MMA. The murine model of MMA harbors a deletion of exon 3 in the Mut gene and has previously been described. $\mathrm{Mut}^{-/-}$ mice on a mixed $(\mathrm{C} 57 \mathrm{BL} / 6 \times 129 \mathrm{~Sv} / \mathrm{Ev} \times \mathrm{FvBN})$ background exhibit a neonatal lethal phenotype, with most mice perishing in the first few days of life $(34,53)$.
Histology. Liver and HCC samples were fixed in formalin, embedded, stained with hematoxylin and eosin, and reviewed by a veterinary pathologist.

$A A V$ production and delivery. The AAV genomes depicted in Figure 1A were packaged into AAV2, AAV8, or AAV9 capsids; purified by cesium chloride centrifugation; and titered by qPCR as previously described (48) by the University of Pennsylvania Vector Core. Viral particles were suspended phosphate-buffered saline and delivered using 32-gauge needle in a total volume of 10 to 20 microliters. Pups received either an intrahepatic or retro-orbital injection of AAV within 24 hours of birth.

Integration mapping and annotation. Samples were prepared for high-throughput sequencing as previously described (54), with the following modifications. The following primers were used for the first round of PCR: AAV ITR primer, 5'-GGAGTTGGCCACTCCCTCTCTG-3', and linker primer, 5'-GTAATACGACTCACTATAGGGCACGCGTG-3'. Cycle conditions were as follows: $95^{\circ} \mathrm{C}$ for 2 minutes and 25 cycles of $95^{\circ} \mathrm{C}$ for 15 seconds, $55^{\circ} \mathrm{C}$ for 30 seconds, and $72^{\circ} \mathrm{C}$ for 1 minute. PCR amplicons from the first round were diluted 1:50. The following primers were used for the second round of PCR: AAV ITR nested primer, 5'-TCTCTGCGCGCTCGCTCG-3', and nested linker primer, 5'-GCGTGGTCGACTGCGCAT- $3^{\prime}$. Cycle conditions were as follows: $95^{\circ} \mathrm{C}$ for 2 minutes and 20 cycles of $95^{\circ} \mathrm{C}$ for 15 seconds, $58^{\circ} \mathrm{C}$ for 30 seconds, and $72^{\circ} \mathrm{C}$ for 1 minute.

The LM-PCR amplicon library was sequenced on an Illumina MiSeq. Integration sites were detected using AAV_GeIST, a modification of the GeIST workflow described in LaFave et al. (29). The workflow continued to use BamTools version 1.0.2, cutadapt version 0.9.3., and Bowtie version 0.12.7 (55-57). The major modifications were as follows. Barcodes were used to identify discrete tissue samples rather than flasks of cells. Each sample received 2 barcodes. ITR trimming occurred in 2 steps in order to accommodate the variable length of the AAV sequence at the integration junction. First, sequences that matched the nested ITR primer (5'-TCTCTGCGCGCTCGCTCG-3') were trimmed by cutadapt (57). Second, the variable region was removed by a Perl script that trimmed the read for as long as consecutive bases matched a continuous string within the variable portion of the AAV ITR (58). If an ITR primer had been detected, the continuous string was required to be directly adjacent to the ITR.

We discarded the 2,466 putative integrations that mapped within the Mut gene (chr17:40,934,684-40,961,989), because the exons of this sequence were carried on the AAV vector and could have represented false positives arising from vector amplification. In addition, direct PCR amplification of a subset of samples with the highest fragment count failed to verify AAV integrations in the Mut locus. Integrations were annotated using Mus musculus genes (Genome Reference Consortium Mouse Build 38 patch release 1) from Ensembl Genes 70, downloaded from BioMart $(59,60)$. Integrations that fell within transcripts were annotated with respect to the nearest exon.

The raw data are available in the NCBI Sequence Read Archive (BioProject accession PRJNA246494). The mapping and annotation scripts we used, along with associated Perl files, as well as hosts files indicating the position of the integrations, the annotations, and a list indicating which sample was in each well are available in Supplemental File Descriptions. 
Targeted PCRs and quantification of AAV integrations. DNA was extracted from livers and HCCs using DNeasy (Qiagen). PCRs were performed using 250 nanograms of genomic DNA as follows. The following primers were used: AAV reverse primer, 5'-CCATTACCCTGGTAGATAAGTAGC-3', and Mir341-5' forward, 5'-GCTTGAAGTGAGGACTGAAGC-3', or Mir341-3' reverse, 5'-GCTGGGAGACTGTGAGTATTG-3', or AC-5' forward, 5'-TCTCACCAAGATCAAAGGCTTCC-3', or AC-3' reverse, 5'-ATGAGAAGTTTAGCCCCCAAAGG-3'. Cycle conditions were as follows: $95^{\circ} \mathrm{C}$ for 2 minutes and 30 cycles of $95^{\circ} \mathrm{C}$ for 15 seconds, $60^{\circ} \mathrm{C}$ for 30 seconds, and $72^{\circ} \mathrm{C}$ for 1 minute.

The amplification products were isolated and subjected to Sanger sequencing. Mouse BLAT $(61,62)$ (assembly December 2011 Genome Reference Consortium Mouse Build 38/mm10) was used to align the resultant sequence to the murine genome.

Standards for copy number were made by serial dilution using a purified common integration fragment and spiked with 250 nanograms of liver genomic DNA extracted from AAV-treated mice that did not develop HCC. PCRs were performed using the AAV reverse and Mir341-5' forward primers and the PCR conditions described above. ImageJ software (http://imagej.nih.gov/ij) was used to created a standard curve and approximate the copy number of the integrations in Mir341.

Microarray processing and analysis. Total RNA and miRNA were extracted using RNeasy and miRNeasy (Qiagen), respectively. Samples were prepared according to Affymetrix protocols (Affymetrix Inc.). RNA quality and quantity were ensured using the Bioanalyzer (Agilent Inc.) and NanoDrop (Thermo Scientific Inc.), respectively. Per RNA labeling, 200 nanograms of total RNA was used in conjunction with the Affymetrix-recommended protocol for the GeneChip 2.0 ST chips. The hybridization cocktail containing the fragmented and labeled cDNAs was hybridized to the Affymetrix Mouse Genome ST 2.0 GeneChip. The chips were washed and stained by the Affymetrix Fluidics Station using the standard format and protocols as described by Affymetrix. The probe arrays were stained with streptavidin phycoerythrin solution (Molecular Probes) and enhanced by using an antibody solution containing $0.5 \mathrm{mg} / \mathrm{ml}$ biotinylated anti-streptavidin (Vector Laboratories). An Affymetrix Gene Chip Scanner 3000 was used to scan the probe arrays. Gene expression intensities were cal- culated using Affymetrix AGCC software. Partek Genomic Suite was used to normalize (Robust Multichip Analysis), summarize, and log transform the data as well as run ANOVA analysis and perform hierarchical clustering. Microarray data are available at the Gene Expression Omnibus (accession GSE57597 and GSE61632; http://www. ncbi.nlm.nih.gov/geo/query/acc.cgi?token=eranyesanvebhsz\&acc=GSE57597 and http://http://www.ncbi.nlm.nih.gov/geo/query/ acc.cgi? token $=$ qnkfiwiezrghdyl\&acc=GSE61632).

Quantitative real-time PCR. Total RNA was extracted from AAVtreated livers and HCCs using the RNeasy Mini Kit (Qiagen). qPCR was accomplished with TaqMan gene expression assays (Gapdh [4352932E], Rtl1 [Mm02392620_s1], and Mir543 [Mm04238293_s1] from Applied Biosystems). Samples were analyzed using an Applied Biosystems StepOnePlus Real-Time PCR System, in accordance with the manufacturer's protocol. All samples were analyzed in triplicate. ABI PRISM Sequence Detection System software was used to calculated $C_{t}$ values. Four individual AAV-treated normal livers were used to determine the relative expression of Rtl1 or Mir543 normalized to Gapdh.

Statistics. Fisher's exact test (2 tailed) or ANOVA were used to calculate the $P$ values. A $P$ value of less than 0.05 was considered significant.

Study approval. All animal studies were reviewed and approved by the National Human Genome Research Institute Animal User Committee (Bethesda, Maryland, USA).

\section{Acknowledgments}

We thank R.W. Blakesley, A. Young, and the staff of the NIH Intramural Sequencing Center for Sequencing and I. Ginty and S. Hoogstraten-Miller for animal care. R.J. Chandler, M.C. LaFave, G.K. Varshney, N.S. Trivedi, N. Carrillo-Carrasco, J.S. Senac, W. Wu, A.G. Elkahloun, S.M. Burgess, and C.P. Venditti were supported by the Intramural Research Program of the National Human Genome Research Institute, NIH.

Address correspondence to: Charles P. Venditti, Genetics and Molecular Biology Branch, National Human Genome Research Institute, National Institutes of Health, 49 Convent Drive Room 4A18, Bethesda, Maryland 20892-4472, USA. Phone: 301.496.6213; E-mail: venditti@mail.nih.gov.
1. Dismuke DJ, Tenenbaum L, Samulski RJ. Biosafety of recombinant adeno-associated virus vectors. Curr Gene Ther. 2013;13(6):434-452.

2. Asokan A, Schaffer DV, Samulski RJ. The AAV vector toolkit: poised at the clinical crossroads. Mol Ther. 2012;20(4):699-708.

3. Mingozzi F, High KA. Therapeutic in vivo gene transfer for genetic disease using AAV: progress and challenges. Nat Rev Genet. 2011;12(5):341-355.

4. Ginn SL, Alexander IE, Edelstein ML, Abedi MR, Wixon J. Gene therapy clinical trials worldwide to 2012 - an update. J Gene Med. 2013;15(2):65-77.

5. Maguire AM, et al. Safety and efficacy of gene transfer for Leber's congenital amaurosis. $N$ Engl JMed. 2008;358(21):2240-2248.

6. Bainbridge JW, et al. Effect of gene therapy on visual function in Leber's congenital amaurosis. N Engl J Med. 2008;358(21):2231-2239.

7. Nathwani AC, et al. Adenovirus-associated virus vector-mediated gene transfer in hemophilia B. N Engl JMed. 2011;365(25):2357-2365.

8. Gaudet D, et al. Efficacy and long-term safety of alipogene tiparvovec (AAV1-LPLS447X) gene therapy for lipoprotein lipase deficiency: an open-label trial. Gene Ther. 2013;20(4):361-369.

9. Yla-Herttuala S. Endgame: glybera finally recommended for approval as the first gene therapy drug in the European union. Mol Ther. 2012;20(10):1831-1832.

10. Donsante A, et al. AAV vector integration sites in mouse hepatocellular carcinoma. Science. 2007;317(5837):477.

11. Donsante A, et al. Observed incidence of tumorigenesis in long-term rodent studies of rAAV vectors. Gene Ther. 2001;8(17):1343-1346.

12. Bell P, et al. No evidence for tumorigenesis of AAV vectors in a large-scale study in mice. $\mathrm{Mol}$ Ther. 2005;12(2):299-306.
13. $\mathrm{Li} \mathrm{H}$, et al. Assessing the potential for AAV vector genotoxicity in a murine model. Blood. 2011;117(12):3311-3319.

14. Montini E. Quest for safety at AAValon. Blood. 2011;117(12):3249-3250.

15. Valdmanis PN, Lisowski L, Kay MA. rAAV-mediated tumorigenesis: still unresolved after an AAV assault. Mol Ther. 2012;20(11):2014-2017.

16. Rosas LE, Grieves JL, Zaraspe K, La Perle KM, Fu H, McCarty DM. Patterns of scAAV vector insertion associated with oncogenic events in a mouse model for genotoxicity. Mol Ther. 2012;20(11):2098-2110.

17. Bell $\mathrm{P}$, et al. Analysis of tumors arising in male B6C3F1 mice with and without AAV vector delivery to liver. Mol Ther. 2006;14(1):34-44.

18. Koeberl DD. Vector-related tumorigenesis not found in ornithine transcarbamylase-deficient mice. Mol Ther. 2006;14(1):1-2. 
19. Kay MA. AAV vectors and tumorigenicity. Nat Biotechnol. 2007;25(10):1111-1113.

20. Zhong L, et al. Recombinant adeno-associated virus integration sites in murine liver after ornithine transcarbamylase gene correction. Hum Gene Ther. 2013;24(5):520-525.

21. Gauttier V, et al. No tumour-initiating risk associated with scAAV transduction in newborn rat liver. Gene Ther. 2013;20(7):779-784.

22. Russell DW. AAV vectors, insertional mutagenesis, and cancer. Mol Ther. 2007;15(10):1740-1743.

23. Hatada I, Morita S, Obata Y, Sotomaru Y, Shimoda $\mathrm{M}$, Kono T. Identification of a new imprinted gene, Rian, on mouse chromosome 12 by fluorescent differential display screening. JBiochem. 2001;130(2):187-190.

24. Luk JM, et al. DLK1-DIO3 genomic imprinted microRNA cluster at $14 \mathrm{q} 32.2$ defines a stemlike subtype of hepatocellular carcinoma associated with poor survival. J Biol Chem. 2011;286(35):30706-30713.

25. Chandler RJ, Venditti CP. Long-term rescue of a lethal murine model of methylmalonic acidemia using adeno-associated viral gene therapy. Mol Ther. 2010;18(1):11-16.

26. Carrillo-Carrasco N, Chandler RJ, Chandrasekaran S, Venditti CP. Liver-directed recombinant adeno-associated viral gene delivery rescues a lethal mouse model of methylmalonic acidemia and provides long-term phenotypic correction. Hum Gene Ther. 2010;21(9):1147-1154.

27. Senac JS, Chandler RJ, Sysol JR, Li L, Venditti CP. Gene therapy in a murine model of methylmalonic acidemia using rAAV9-mediated gene delivery. Gene Ther. 2012;19(4):385-391.

28. Chandler RJ, Venditti CP. Pre-clinical efficacy and dosing of an AAV8 vector expressing human methylmalonyl-CoA mutase in a murine model of methylmalonic acidemia (MMA). Mol Genet Metab. 2012;107(3):617-619.

29. LaFave MC, Varshney GK, Gildea DE, Wolfsberg TG, Baxevanis AD, Burgess S. MLV integration site selection is driven by strong enhancers and active promoters. Nucleic Acids Res. 2014;42(7):4257-4269.

30. Nakai H, Montini E, Fuess S, Storm TA, Grompe M, Kay MA. AAV serotype 2 vectors preferentially integrate into active genes in mice. Nat Genet. 2003;34(3):297-302.

31. Riordan JD, et al. Identification of rtl1, a retrotransposon-derived imprinted gene, as a novel driver of hepatocarcinogenesis. PLoS Genet. 2013;9(4):e1003441.

32. Wang PR, Xu M, Toffanin S, Li Y, Llovet JM, Russell DW. Induction of hepatocellular carcinoma by in vivo gene targeting. Proc Natl Acad Sci U S A. 2012;109(28):11264-11269.
33. Manno CS, et al. Successful transduction of liver in hemophilia by AAV-Factor IX and limitations imposed by the host immune response. Nat Med. 2006;12(3):342-347.

34. Chandler RJ, et al. Mitochondrial dysfunction in mut methylmalonic acidemia. FASEB J. 2009;23(4):1252-1261.

35. Wilnai Y, Enns GM, Niemi AK, Higgins J, Vogel $\mathrm{H}$. Abnormal hepatocellular mitochondria in methylmalonic acidemia. Ultrastruct Pathol. 2014;38(5):309-314.

36. Jeganathan K, Malureanu L, Baker DJ, Abraham SC, van Deursen JM. Bub1 mediates cell death in response to chromosome missegregation and acts to suppress spontaneous tumorigenesis. JCell Biol. 2007;179(2):255-267.

37. Mahler JF, Stokes W, Mann PC, Takaoka M, Maronpot RR. Spontaneous lesions in aging FVB/N mice. Toxicol Pathol. 1996;24(6):710-716.

38. Nakamura K, Kuramoto K, Shibasaki K, Shumiya S, Ohtsubo K. [Age-related incidence of spontaneous tumors in SPF C57BL/6 and BDF1 mice]. Jikken Dobutsu. 1992;41(3):279-285.

39. Huser D, Gogol-Doring A, Chen W, Heilbronn R. Adeno-associated virus type 2 wild-type and vector-mediated genomic integration profiles of human diploid fibroblasts analyzed by third-generation PacBio DNA sequencing. J Virol. 2014;88(19):11253-11263.

40. Li C, Yu S, Zhong X, Wu J, Li X. Transcriptome comparison between fetal and adult mouse livers: implications for circadian clock mechanisms. PLoS One. 2012;7(2):e31292.

41. Dupuy AJ, et al. A modified sleeping beauty transposon system that can be used to model a wide variety of human cancers in mice. Cancer Res. 2009;69(20):8150-8156.

42. Ranzani M, et al. Lentiviral vector-based insertional mutagenesis identifies genes associated with liver cancer. Nat Methods. 2013;10(2):155-161.

43. Themis M, et al. Oncogenesis following delivery of a nonprimate lentiviral gene therapy vector to fetal and neonatal mice. Mol Ther. 2005;12(4):763-771.

44. Nowrouzi A, et al. The fetal mouse is a sensitive genotoxicity model that exposes lentiviral-associated mutagenesis resulting in liver oncogenesis. Mol Ther. 2013;21(2):324-337.

45. Hacein-Bey-Abina S, et al. A serious adverse event after successful gene therapy for X-linked severe combined immunodeficiency. N Engl J Med. 2003;348(3):255-256.

46. Barzel A, et al. Promoterless gene targeting without nucleases ameliorates haemophilia $\mathrm{B}$ in mice [published online ahead of print October 29, 2014]. Nature. doi:10.1038/nature13864.

47. McCarty DM. Self-complementary AAV vec- tors; advances and applications. Mol Ther. 2008;16(10):1648-1656.

48. Gao GP, Alvira MR, Wang L, Calcedo R, Johnston J, Wilson JM. Novel adeno-associated viruses from rhesus monkeys as vectors for human gene therapy. Proc Natl Acad Sci US A. 2002;99(18):11854-11859.

49. Chen YH, Claflin K, Geoghegan JC, Davidson BL. Sialic acid deposition impairs the utility of AAV9, but not peptide-modified AAVs for brain gene therapy in a mouse model of lysosomal storage disease. Mol Ther. 2012;20(7):1393-1399.

50. Bantel-Schaal U, Hub B, Kartenbeck J. Endocytosis of adeno-associated virus type 5 leads to accumulation of virus particles in the Golgi compartment. J Virol. 2002;76(5):2340-2349.

51. Thomas CE, Storm TA, Huang Z, Kay MA. Rapid uncoating of vector genomes is the key to efficient liver transduction with pseudotyped adeno-associated virus vectors. J Virol. 2004;78(6):3110-3122.

52. Salganik M, Aydemir F, Nam HJ, McKenna R, Agbandje-McKenna M, Muzyczka N. Adeno-associated virus capsid proteins may play a role in transcription and second-strand synthesis of recombinant genomes. J Virol. 2014;88(2):1071-1079.

53. Chandler RJ, et al. Metabolic phenotype of methylmalonic acidemia in mice and humans: the role of skeletal muscle. BMC Med Genet. 2007;8:64.

54. Varshney GK, et al. A large-scale zebrafish gene knockout resource for the genome-wide study of gene function. Genome Res. 2013;23(4):727-735.

55. Barnett DW, Garrison EK, Quinlan AR, Stromberg MP, Marth GT. BamTools: a C++ API and toolkit for analyzing and managing BAM files. Bioinformatics. 2011;27(12):1691-1692.

56. Langmead B, Trapnell C, Pop M, Salzberg SL. Ultrafast and memory-efficient alignment of short DNA sequences to the human genome. Genome Biol. 2009;10(3):R25.

57. Martin M. Cutadapt removes adapter sequences from high-throughput sequencing reads. EMBnetJ. 2011;17:(1).

58. Linden RM, Ward P, Giraud C, Winocour E, Berns KI. Site-specific integration by adeno-associated virus. Proc Natl Acad Sci U S A 1996;93(21):11288-11294.

59. Flicek P, et al. Ensembl 2013. Nucleic Acids Res. 2013;41(Database issue):D48-D55.

60. Kinsella RJ, et al. Ensembl BioMarts: a hub for data retrieval across taxonomic space. Database (Oxford). 2011;2011:bar030.

61. Kent WJ. BLAT - the BLAST-like alignment tool. Genome Res. 2002;12(4):656-664.

62. Kent WJ, et al. The human genome browser at UCSC. Genome Res. 2002;12(6):996-1006. 